\title{
Estados afectivos emocionales (depresión, ansiedad y estrés) en personal de enfermería del sector salud pública de México'
}

\section{Emotional affective states (depression, anxiety and stress) of nursing in a mexican public health sector}

\author{
Blanca García-Rivera ${ }^{2}$ Sonia Maldonado-Radillo \\ María Ramírez Barón \\ Universidad Autónoma de Baja California, México
}

(Rec: septiembre 2013 - Acep: junio 2014)

\section{Resumen}

Este trabajo es una investigación de tipo descriptivo-correlacional, transversal con diseño no experimental sobre el estado afectivo emocional de los trabajadores de la Salud. Su objetivo es identificar la existencia de diferencias significativas entre el género y estados afectivos emocionales (depresión, ansiedad y estrés) de los trabajadores de una institución del sector salud pública ubicada en la ciudad de Ensenada perteneciente al estado de Baja California situado al noroeste de México. Se trabajó con una muestra por conveniencia la cual integra a 130 empleados; en la recolección de los datos se utilizó la escala DASS-21. Los resultados identificaron los estados afectivos emocionales dentro de un rango normal y que no existen diferencias estadísticamente significativas en cuanto al género; además, se encontraron correlaciones fuertes, positivas y significativas entre las subescalas del DASS-21.

Palabras clave: depresión, ansiedad, estrés, enfermeras.

\begin{abstract}
This study is a descriptive correlational cross-sectional approach with a non-experimental design about emotional affective states related dissorders in Mexican public health workers. It's goal was to identify the existence of significant differences between gender and emotional affective state (depression, anxiety and stress) for workers in a hospital located in Ensenada, Baja California in north-western Mexico. The work covers a sample of 130 employees. To collection of data used a DASS-21 scale. The results identified an emotional affective state within a normal range. No statistically differences in gender were identified. Positive and significant correlations between subscales of the DASS-21 were found.
\end{abstract}

Keywords: depression, anxiety, stress, nurses.

\footnotetext{
Este artículo está asociado al proyecto "Compassion satisfaction, compassion fatigue, burnout, depression, anxiety and stress in nurses employed at SCGH", No 207528, apoyado por el Consejo Nacional de Ciencia y Tecnología (CONACYT).

2 Correspondencia dirigida a: Blanca Rosa García-Rivera, Blvd Zertuche S/N, Ensenada, B.C. Teléfono y Fax: (646) 1766600 Ext. 133. Correo electrónico: blanca_garcia@uabc.edu.mx.
} 


\section{Introducción}

Los cambios económicos, políticos y tecnológicos de los últimos 30 años han repercutido de manera directa sobre los procesos laborales y el diseño del trabajo, originando riesgos psicosociales que deterioran la salud de los trabajadores afectando su desempeño laboral e incluso, su calidad de vida (Gil-Monte, 2010).

Esta situación es frecuente entre los profesionales del sector salud los cuales desarrollan su trabajo entre la vida y la muerte, generalmente laboran bajo presión, en confinamiento, teniendo un contacto directo con el usuario y sus padecimientos; circunstancias que aumentan el riesgo de padecer algún trastorno de sus estados afectivos emocionales (Abreu, 2011); esto como consecuencia al involucramiento de sus sentimientos en las enfermedades de otros, lo cual constituye un proceso desgastante que, en algunas ocasiones, puede presentar alteraciones principalmente del humor donde se manifiesta como depresión o irritabilidad (De Vargas \& Dias, 2011) y ansiedad.

Estas manifestaciones aplican al trabajo de enfermería, el cual ha sido considerado como una profesión de prevalencias altas de trastornos psicológicos que, en cierta forma, repercuten negativamente en su salud y calidad de vida provocando ausentismo laboral y afectación en la calidad de su trabajo (Lerma-Martínez, Rosales-Arellano \& Gallegos-Alvarado, 2009).

Pero, sin duda, uno de esos trastornos psicológicos que acapara la atención es el estrés laboral; un problema de gran magnitud y trascendencia mundial, afecta al empleado y la productividad en el ámbito hospitalario. Este es considerado como la segunda causa de baja laboral y aunque los costos por estrés en el trabajo son difíciles de evaluar, se sabe que la Unión Europea estima que, anualmente, 40 millones de empleados lo padecen suponiendo un costo de alrededor de 20 . 000 millones de euros, excluyendo las pérdidas por productividad (Enríquez, Colunga, Preciado, Ángel \& Domínguez, 2011).

El estrés es un fenómeno sumamente complejo, incluso entre los investigadores existen controversias en torno a este término por lo que no han conseguido crear una definición que satisfaga a todos; de hecho, existen definiciones en las que se considera como un estímulo, otras que están centradas en la respuesta producida en el organismo y, en tercer lugar, las definiciones de tipo interactivo o transaccional. Aunque con el transcurso del tiempo este ha sido abordado desde un modelo biopsico-social integrador en el que el enfoque psicológico aportó definiciones del estrés como estímulo, como respuesta y/o como interacción entre ambos (Sierra, Ortega \& Zubeidat, 2003).

Debido a las alteraciones que el estrés provoca en la persona, se puede entender este concepto como: "un conjunto de reacciones fisiológicas y psicológicas que experimenta el organismo cuando se lo somete a fuertes demandas" (Melgosa, 1995, p. 19). Este mismo autor asegura que el estrés no sobreviene de manera repentina, desde su aparición hasta que alcanza su máximo efecto, pasa por tres etapas: alarma, resistencia y agotamiento.; además, con mucha frecuencia la persona estresada presenta síntomas de ansiedad, tales como la aprehensión, la preocupación, la tensión y el temor por el futuro (Naranjo, 2009).

De acuerdo con Barco, Miranda, Herrera y Álvarez (2008), el estrés se presenta en un individuo cuando le exigen un rendimiento superior al normal, lo cual lo pone en riesgo de enfermar. En este sentido, el estrés laboral se debe considerar como el producto de la relación entre una persona y su entorno laboral. En este tipo de situaciones, el individuo emite una respuesta de estrés que consiste en el aumento de la activación fisiológica y cognitiva del organismo, que al mismo tiempo, se prepara para una intensa actividad motora.

En igual forma, la depresión y la ansiedad son trastornos del estado del ánimo que se presentan en los trabajadores de la salud que, al igual que el estrés, también afectan los resultados organizacionales, tales como: un aumento del ausentismo, antagonismo, falta de compromiso con la organización, insatisfacción en el trabajo o conflictos interpersonales (Flores-Villavicencio, Troyo-Sanromán, Valle \& Vega-López, 2010).

La depresión es tanto un estado afectivo normal (tristeza) como un síntoma o un síndrome que se caracteriza por alteraciones del humor (irritabilidad, falta de capacidad de sentir placer, apatía), cognitivas, psicomotoras y vegetativas (sueño y apetito) (De Vargas \& Dias, 2011). A diferencia de estado anterior, la ansiedad se ubica solo como estado emocional que se caracteriza por la aprensión y tensión, que incluye sensaciones de nerviosismo y preocupación. Su nivel no depende tanto de la situación estresora como de la propia percepción del empleado sobre la situación (Ramis, Torregrosa, Viladrich, \& Cruz, 2010).

En el contexto referido, la depresión es un término que proviene de una raíz latina: depressio, que significa opresión o abatimiento. La literatura aporta diversas investigaciones (Korman \& Sarudiansky, 2011) en donde se realiza una revisión profunda sobre modelos teóricos y clínicos para la conceptualización de la depresión; un abordaje sencillo es considerarla como 
un trastorno del estado de ánimo caracterizado por una sensación de tristeza intensa superior a dos meses (Piqueras, et al., 2008).

Las causas que producen la depresión son múltiples, entre las que figuran: acontecimientos de la vida diaria, cambios químicos en el cerebro, efecto secundario de medicamentos, diversos trastornos físicos o médicos (Piqueras, et al., 2008). Estos mismos autores consideran que la depresión "es el resultado final de la interacción de múltiples factores constitucionales, evolutivos, ambientales e interpersonales, que modifican las pautas de neurotransmisión entre los hemisferios cerebrales y el sistema límbico".

Por lo que refiere a la ansiedad, ésta ha evolucionado hacia una multiplicidad de dimensiones; en el principio de su conceptualización, fue considerada como un estado emocional y fisiológico transitorio, como un rasgo de personalidad y como explicación de una conducta. En la última década del siglo pasado, ese concepto se concibe como estado emocional que se da solo o sobreañadido a los estados depresivos y a los síntomas psicosomáticos cuando el sujeto fracasa en su adaptación al medio (Sierra, et al., 2003).

El concepto de ansiedad alude a un estado de agitación e inquietud desagradable caracterizado por la anticipación del peligro, el predominio de síntomas psíquicos y la sensación de catástrofe o de peligro inminente (Sierra, et al., 2003). La ansiedad puede ser definida como una respuesta del organismo que se desencadena ante una situación de amenaza o peligro físico o psíquico, cuyo objeto es dotar al organismo de energía para anular o contrarrestar el peligro mediante una respuesta (Sandín \& Chorot, 1995, en Piqueras et al., 2008).

Melgosa (1995) considera que existe una estrecha vinculación entre estrés y ansiedad, pues muchos individuos, que en la actualidad sufren los efectos devastadores de la ansiedad, iniciaron sus hábitos ansiosos en medio de situaciones estresantes (p. 40). Bensabat (1987, citado en Naranjo, 2009) describe la ansiedad como una emoción de estrés, confundiendo a la misma con el estado agudo de este.

A finales de la década de 1970, aparecieron los primeros estudios sobre profesiones relacionadas con la atención a pacientes (enfermería, medicina, etc.); dentro de éstos destacan investigaciones sobre el síndrome de desgaste profesional (burnout) y las reacciones emocionales vinculadas con el estrés, la depresión y ansiedad, las cuales giraron en torno al agotamiento personal como resultado de las actividades propias de la prolongada prestación de servicios y cuidado de personas con problemas de salud (Hurtado \& Pereira,
2012). Al respecto, Garcés, (2002) citado en Miño (2012) sostiene que el síndrome también llamado estrés laboral, se presenta debido a que las requerimientos del trabajo exceden los recursos que la persona posee para dicha actividad.

A nivel internacional se tiene evidencia de numerosas investigaciones sobre los niveles emocionales del personal del sector salud (Abreu, 2011; Alves, Alves \& Pineda, 2007; López, Zegarra \& Cuba, 2006; Martínez, Centeno, Sanz-Rubiales \& Del Valle, 2009). En México existen pocos estudios reportados (FloresVillavicencio, et al., 2010; Enríquez, et al., 2011) y casi ninguno, en lo que refiere al estado de Baja California ya que únicamente se localizó una investigación realizada en Tijuana, B.C. por Hernández (2005), en la cual se estudia la depresión en los niños abordando sus tipos, síntomas, causas y terapias; ésta concluye que se trata de un padecimiento más frecuente de lo que se piensa $\mathrm{y}$ de lo que en realidad se diagnostica.

Considerando este vacío de información y que el sistema de salud pública de México presenta una problemática muy grave por la falta de recursos y el creciente número de pacientes que demandan atención médica, es imprescindible dirigir la atención a la evaluación los factores que inciden en la salud del tipo de profesionales que atiende a varios millones y millones de mexicanos. Tener conocimiento sobre su salud facilitará el establecimiento de acciones que reduzcan estos padecimientos, contribuyendo así a un desempeño eficiente de sus labores.

Esta investigación tiene como propósito evaluar el estado afectivo emocional de los trabajadores del sector de salud pública y su objetivo es identificar la existencia de diferencias significativas entre el género y los estados afectivos emocionales (depresión, ansiedad y estrés) de los trabajadores de una institución del sector salud pública ubicada en la ciudad de Ensenada perteneciente al estado de Baja California (B. C.) situado al noroeste de la República Mexicana (México). Teniendo como objetivos específicos: (1) Conocer el nivel de los estados emocionales afectivos (estrés, depresión y ansiedad) de los trabajadores del sector salud pública de México; (2) determinar la existencia de asociación entre las subescalas del DASS-21 (estrés, depresión y ansiedad), (3) Identificar diferencias entre los estados afectivos emocionales de los trabajadores y su género. Por último, con la realización de este trabajo se pretende aportar nuevos conocimientos acerca de la depresión, ansiedad y estrés que prevalece entre los empleados del sector salud pública de la región de Baja California en México; estos conocimientos proporcionan elementos 
importantes para el diseño de estrategias de prevención adecuadas a las necesidades del colectivo de trabajadores del sector estudiado.

\section{Método}

Esta investigación tiene un enfoque cuantitativo cuyo diseño es no experimental, específicamente transversal, identificado como descriptivo-correlacional; descriptivo por tener la intención de especificar las características de los estados afectivos emocionales (Depresión, Ansiedad y Estrés) de los empleados del sector salud pública y correlacional por tener la finalidad de identificar la probable existencia de asociación entre las variables.

\section{Participantes y tipo de muestreo}

La población de estudio se constituye por empleados del Instituto de Seguridad y Servicios Sociales de los Trabajadores del Estado de Baja California (B.C.) perteneciente a la región noroeste de la República Mexicana, el hospital está localizado en la ciudad de Ensenada, B.C. Por la naturaleza de las actividades de la propia institución se decidió por un muestreo por conveniencia (Casal \& Mateu, 2003), seleccionando a 130 empleados con características similares a la población y cuyo perfil sociodemográfico se detalla en la Tabla 1.

\section{Instrumento de medición}

En este estudio, se emplea la Escala de Depresión, Ansiedad y Estrés (DASS-21). La subescala de Depresión considera aspectos relacionados con el bajo afecto positivo como la disforia, desesperanza, tristeza o anhedonia. En cambio, la subescala Ansiedad evalúa aspectos relacionados con la activación psicofisiológica o la excitación autonómica (sudor de manos, temblor, etc.), y experiencias subjetivas de ansiedad. Por su parte, la subescala Estrés, evalúa la dificultad para estar relajado, la excitación nerviosa, la agitación, la irritabilidad y la impaciencia.

Por lo que refiere al instrumento utilizado, éste es un paquete de tres escalas (de 7 ítems cada una) de tipo Likert, de cuatro puntos de severidad/frecuencia para medir la extensión de cuales estados han experimentado durante la semana pasada. El DASS-21 se basa en una dimensional en lugar de una concepción categórica de trastorno psicológico. La premisa en la que se basa el DASS-21 (y que fue confirmado por los datos de la investigación) es que las diferencias entre la depresión, ansiedad y estrés experimentados por sujetos normales y por los que están perturbados en la clínica, son esencialmente diferencias de grado (Lovibond \& Lovibond, 1995).

En este estudio se comprobó la fiabilidad del instrumento mediante el cálculo del alfa de Cronbach el cual se utiliza para evaluar la homogeneidad de los distintos ítems de una misma variable. El resultado fue un alfa de Cronbach (global) de 0.911; para depresión un $\alpha=0.758$, para ansiedad $\alpha=0.732$ y para estrés $a=0.826$.

\section{Procedimiento}

La técnica de recogida de los datos fue un cuestionario autoadministrado que se aplicó a una muestra de los trabajadores de la institución (incluyendo los tres turnos de trabajo). La aplicación se efectuó durante los meses de febrero, marzo y abril del 2013 en el hospital en donde desempeñan sus labores, el cual se ubica en la ciudad de Ensenada, B.C. México.

\section{Tratamiento de datos}

En el tratamiento estadístico de la información se utilizó el paquete Statistical Package for the Social Siences (SPSS) ® las pruebas estadísticas utilizadas en el estudio fueron: U de Mann-Whitney, correlaciones de Spearman, estadísticas de resumen y frecuencias. Dado que las variables depresión ansiedad y estrés no presentan distribución normal se optó por el uso de pruebas no paramétricas.

Los valores de la escala DASS-21 se calcularon multiplicando por dos cada subescala; para cada estado se empleó la tipología: normal, leve, moderado, severo y severo extremo. La determinación de la correlación entre las subescalas (depresión, ansiedad y estrés) se realizó a través del cálculo del coeficiente rho de Spearman.

\section{Resultados}

\section{Perfil sociodemográfico}

Según el género, el $86.15 \%$ de los empleados eran mujeres y $13.85 \%$ hombres; el rango etario de la mayoría corresponde al $50.76 \%$ de los respondientes y es entre los 
23 y 32 años de edad; respecto al estado civil se encontró que el $47.7 \%$ eran solteros, el $43.7 \%$ casado o vivían en unión consensual, $6.2 \%$ separados y $1.5 \%$ divorciados. Con relación a la antigüedad en la institución, el 55.38\% tienen una antigüedad menor a 3 años, el $30.76 \%$ entre 4 y 9 años, el $10.76 \%$ ha trabajado 10 años o más y el $3.07 \%$ no respondieron la pregunta. Respecto al turno de trabajo, el $39.23 \%$ trabajaban en turno matutino, $36.92 \%$ en turno vespertino, $6.15 \%$ turno nocturno, y el $17.69 \%$ tiene un turno mixto (Ver tabla 1).

\section{Nivel de depresión, ansiedad y estrés}

Todos los estados afectivos emocionales se encontraron en un nivel normal; $78.5 \%$ para la depresión, $71.5 \%$ para la ansiedad y $74.6 \%$ para el estrés. Como puede observarse en la Tabla 2, solamente el $21.5 \%$ de los individuos presentan cierto grado de depresión, la cual no fue detectada en nivel severo extremo. Por lo que refiere a la ansiedad, aparece en $28.5 \%$ del personal y de éstos el $4.6 \%$ la manifiesta en grado

Tabla 1

Perfil demográfico de los empleados

\begin{tabular}{llcc}
\hline Característica & & Frecuencia & Porcentaje (\%) \\
\hline \multirow{2}{*}{ Sexo } & Femenino & 112 & 86.15 \\
& Masculino & 18 & 13.85 \\
Edad (Años) & Hasta 22 años & 15 & 11.53 \\
& Entre 23 y 32 años & 66 & 50.76 \\
& Entre 33 y 42 años & 44 & 33.84 \\
& Más de 43 años & 5 & 3.84 \\
Estado civil & Soltero & 62 & 47.7 \\
& Casado & 53 & 41.4 \\
& Conviviente & 3 & 2.3 \\
& Separado & 8 & 6.2 \\
Antigüedad en la & Divorciado & 2 & 1.5 \\
institución (Años) & No especificó & 2 & 1.5 \\
& Menos de 3 años & 72 & 55.38 \\
& Entre 4 y 9 años & 40 & 30.76 \\
Turno de trabajo & No más años & 14 & 10.76 \\
& Matutino & 4 & 3.07 \\
& Vespertino & 51 & 39.23 \\
& Nocturno & 48 & 36.92 \\
& Mixto & 23 & 6.15 \\
& Fucó & & 17.69 \\
\hline
\end{tabular}

Fuente: Elaboración propia con datos del trabajo de campo

Tabla 2

Niveles de los estados afectivos emocionales de los individuos

\begin{tabular}{|c|c|c|c|c|c|c|}
\hline \multirow{2}{*}{$\begin{array}{l}\text { Estados Afectivos } \\
\text { Emocionales }\end{array}$} & \multicolumn{6}{|c|}{$\begin{array}{c}\text { Niveles de los Estados Afectivos Emocionales } \\
\text { (Frecuencias expresadas en \%) }\end{array}$} \\
\hline & Normal & Leve & Moderado & Severo & $\begin{array}{l}\text { Severo } \\
\text { extremo }\end{array}$ & TOTAL \\
\hline Depresión & $78.5 \%$ & $10.8 \%$ & $8.5 \%$ & $2.2 \%$ & $0 \%$ & $100 \%$ \\
\hline Ansiedad & $71.5 \%$ & $3.8 \%$ & $16.9 \%$ & $3.2 \%$ & $4.6 \%$ & $100 \%$ \\
\hline Estrés & $74.6 \%$ & $13.1 \%$ & $7.7 \%$ & $3.8 \%$ & $0.8 \%$ & $100 \%$ \\
\hline
\end{tabular}

Fuente: Elaboración propia con datos de campo 
severo extremo. En el caso del estrés también se ubican manifestaciones de severidad extrema aunque en un mínimo porcentaje $0.8 \%$.

En un análisis exhaustivo de los niveles de los estados afectivos emocionales (Figuras 1,2 y 3 ) es posible apreciar que en su mayoría, más del $70 \%$ en ambos sexos, se encuentran dentro de un rango normal; no obstante, se detectó que únicamente en las mujeres aparecen niveles severos extremos de ansiedad (5.4\%) y de estrés $(0.9 \%)$, en los hombres no aparecen niveles

Figura 1.

Niveles de Depresión entre sexos

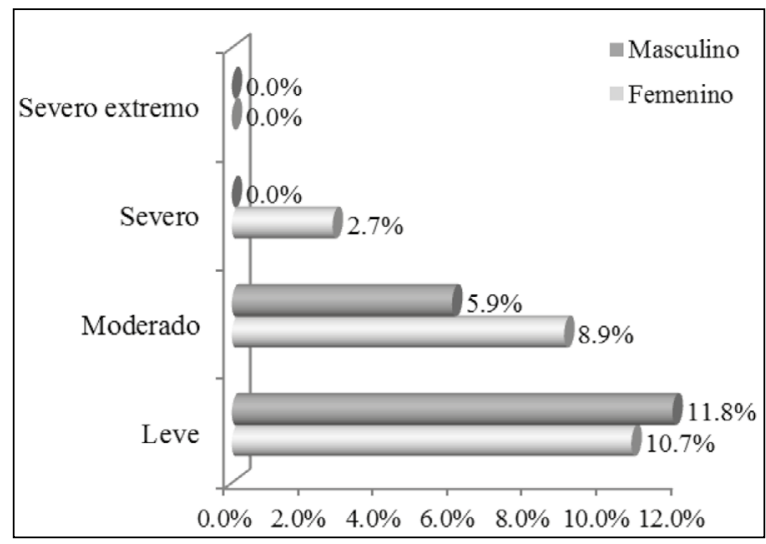

Los cuatro niveles de la Depresión (leve, moderado, severo y severo extremo) se encuentran expresados en porcentaje.

Fuente: Elaboración propia

Figura 2.

Niveles de Ansiedad entre sexos

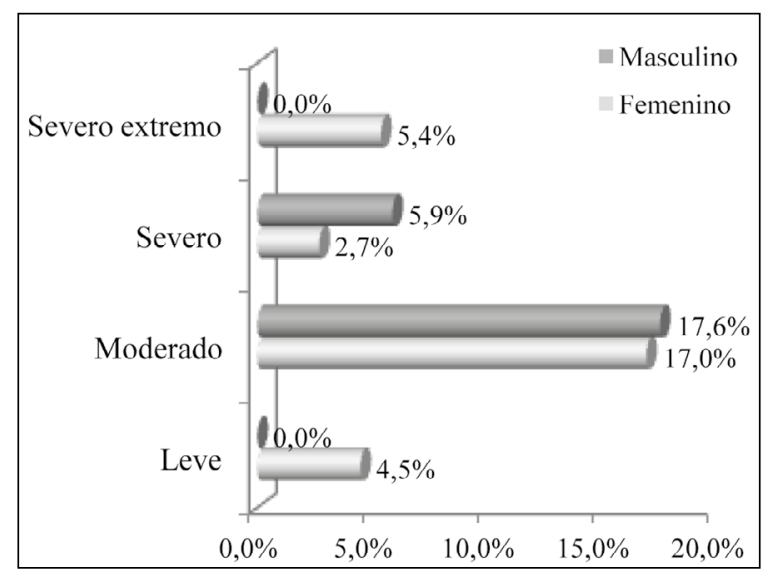

Los cuatro niveles de Ansiedad (leve, moderado, severo y severo extremo) se encuentran expresados en porcentaje.

Fuente: Elaboración propia
Figura 3.

Niveles de Estrés entre sexos

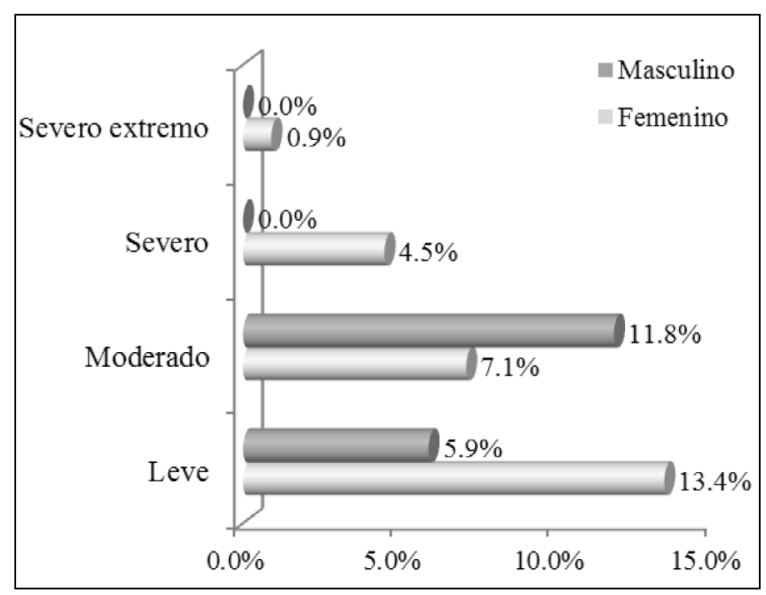

Los cuatro niveles de Estrés (leve, moderado, severo y severo extremo) se encuentran expresados en porcentaje.

Fuente: Elaboración propia

severos extremos de ninguno de los estados afectivos emocionales.

\section{Diferencias en los estados afectivos emocionales de acuerdo al género}

Para identificar las diferencias entre hombres y mujeres respecto a sus manifestaciones de depresión, ansiedad y estrés se utilizó la prueba U-Mann-Witney. Los resultados (Tabla 4) revelan que para la depresión el p-valor asociado al estadístico z (-.513) fue $\geq a$ $(0.608 \geq 0.05)$ por lo que se acepta la hipótesis nula, puesto que en el nivel de depresión al comparar las medias del género se encontró que en éstas las diferencias no son estadísticamente significativas. En términos semejantes también fue lo encontrado para la ansiedad, el p-valor asociado al estadístico z (.-.469) fue $\geq a(0.639 \geq 0.05)$ aceptando también la hipótesis nula, así, en el grado de ansiedad también las diferencias en los grupos no son estadísticamente significativas. Lo mismo fue detectado para el estrés un p-valor asociado al estadístico z (-.704) fue $\geq a(.481 \geq 0.05)$ por lo que de igual manera se acepta la hipótesis nula con lo que se evidencia las no diferencias entre las medias de los dos grupos, las cuales no son estadísticamente significativas. 
DEL SECTOR SALUD PÚBLICA DE MÉXICO

Tabla 4.

Diferencias en los estados afectivos emocionales de los individuos según el género

\begin{tabular}{lccccc}
\hline \multirow{2}{*}{ Estados Afectivos } & Sexo & $\mathrm{N}$ & Mediana & \multicolumn{2}{c}{ U Mann-Whitney } \\
\cline { 5 - 6 } & & & & $\mathrm{z}$ & $\mathrm{p}$-valor \\
\hline \multirow{2}{*}{ Depresión } & Femenino & 112 & 65.47 & -.513 & .608 \\
& Masculino & 17 & 61.88 & & \\
Ansiedad & Femenino & 112 & 65.48 & -.469 & .639 \\
& Masculino & 17 & 61.85 & & .481 \\
Estrés & Femenino & 112 & 65.68 & -.704 & \\
\hline
\end{tabular}

Fuente: Elaboración propia con datos de campo

\section{Relaciones entre subescalas del DASS-21}

Con la intención de averiguar si existe asociación entre las subescalas que forman parte del DASS-21 se utilizó la correlación de Spearman, con la cual se logró identificar que la asociación entre la depresión, ansiedad y estrés resultó estadísticamente significativa. Se encontraron relaciones positivas con un grado de asociación de 68.9\% ( $\mathrm{r}=.689 ; \mathrm{p}<0.05) ; 74.1 \%(\mathrm{r}=.741$; $\mathrm{p}<0.05) ; y, 69.6 \%$ (r=.696; $\mathrm{p}<0.05)$ (Tabla 5). De acuerdo a lo señalado por Martínez et al. (2009), la asociación entre las subescalas es entre moderada y fuerte.

Tabla 5.

Correlación entre las variables del DASS-21

\begin{tabular}{lllll}
\hline Estados Afectivos & Spearman's rho & Depresión & Ansiedad & Estrés \\
\hline \multirow{2}{*}{ Depresión } & Coeficiente de correlación & 1.000 & $.689^{* *}$ & $.741^{* *}$ \\
& p-valor &. & .000 & .000 \\
Ansiedad & Coeficiente de correlación & $.689^{* *}$ & 1.000 & $.696^{* *}$ \\
& p-valor & .000 &. & .000 \\
\multirow{2}{*}{ Estrés } & Coeficiente de correlación & $.741^{* *}$ & $.696^{* *}$ & 1.000 \\
& p-valor & .000 & .000 &. \\
\hline
\end{tabular}

**. Correlaciones significativas a un $\mathrm{p}$ valor de 0.01 (2-colas)

Fuente: Elaboración propia con datos de campo

\section{Discusión}

Antes de realizar el análisis de los datos fue verificada la confiabilidad del cuestionario, encontrando que los niveles de consistencia interna (alfa de Cronbach) para las subescalas de la DASS-21 oscilaron entre .73 y .82 , siendo totalmente coincidentes con los encontrados en otros estudios (Antony et al., 1998; Bados, Solana \& Andrés, 2005; Henry \& Crawford, 2005; Tully, Zajac \& Venning, 2009), por lo que se considera pertinente la aplicación del instrumento.

En esta investigación se identificaron los niveles de depresión, ansiedad y estrés en los trabajadores de la salud; adicionalmente, se analizó la existencia de diferencias estadísticamente significativas entre el género y los estados afectivos emocionales; $y$, si existen asociaciones entre estas subescalas. El resultado principal de este trabajo es que la mayoría $(>70 \%)$ de los participantes de este estudio, tanto hombres como mujeres, posee estados afectivos emocionales (depresión, ansiedad y estrés) dentro de un rango normal.

Este resultado coincide con la investigación realizada por Lerma-Martínez, Rosales-Arellano y Gallegos-Alvarado (2009) en cuyo reporte la depresión y ansiedad también se manifiestan en rango similares; en contraste con lo que ocurre en trabajos con estudiantes de enfermería en donde fueron encontrados valores más altos (Alves, Alves \& Pineda, 2007; Vásquez \& 
Ríos, 2009), lo cual podría ser aceptable dado que los estudiantes aparte de todas las situaciones que implica estar estudiando, tienen la responsabilidad de atender adecuadamente a los pacientes.

Referente a las diferencias entre el género y los niveles de depresión, ansiedad y estrés en los profesionales de enfermería estudiados se encontraron diferencias estadísticas que no son significativas al comparar las medias de los grupos (femenino y masculino); situación que contrasta con el estudio de Hernández (2003) en el cual se encontró que los síntomas de estrés con valores promedio significativamente mayores en las mujeres.

Respecto a la relación entre las subescalas, se encontraron asociaciones entre moderadas y fuertes. La relación más fuerte fue detectada entre la depresión y el estrés $(r=0.741)$ estadísticamente significativa a un p-valor de 0.01; tales resultados son coincidentes con investigaciones previas de (Alves, Figuereido, Cruz \& Alves, 2011; Lovibond \& Lovibond, 1995; Tully, Zajac y Venning, 2009; Zlomke, 2009); sin embargo este hecho puede ser indicativo de la escasa validez discriminante de las subescalas, lo cual deberá comprobarse a través del análisis correspondiente. Autores como Fonseca-Pedrero, Paino, Lemos-Giráldez y Múñiz (2010) opinan que esa fuerte asociación entre las subescalas tiene relación con la naturaleza dimensional y no categórica de los constructos psicológicos.

Los resultados aquí expuestos deberán tomarse con reserva ya que en la investigación la selección de los trabajadores se realizó a través de un muestreo por conveniencia y no se tiene la seguridad de que cada unidad muestral integre a la población total en el proceso de selección de la muestra. Además, estos hallazgos deben interpretarse como una tendencia de la situación, por tanto no informan las razones de los padecimientos; de ahí que, estas particularidades constituyen limitantes a este estudio. No obstante, la información obtenida es un buen marco de referencia para investigaciones posteriores en las cuales sería recomendable estudiar otras variables sociodemográficas, tales como el estado civil, la edad, etc.

\section{Referencias}

Abreu, F. J. (2011). Síndrome de agotamiento emocional en profesionales de la unidad de cuidados intensivos, Revista Multiciencias, 11(4), 370-377. Recuperado de http://www.redalyc.org/articulo. oa?id=90421972006

Alves, J. L.; Alves, M. \& Pineda, J. (2007). Evaluación de los estados emocionales de estudiantes de enfermería. Index de Enfermería, 16(56), 26-29. Recuperado de http://scielo.isciii.es/ scielo.php?pid=S1132-12962007000100006\&script=sci_arttext
Alves, A. J., Figueiredo, M. H., Cruz, A. \& Alves, M. (2011). Depression, Anxiety and Stress in primary health care users. Revista Latino-Americana de Enfermagem (RLAE), 19(2), 348-353. http:// dx.doi.org/10.1590/S0104-11692011000200017

Antony, M. M., Bieling, P. J., Cox, B. J., Enns, M. W. \& Swinson, R. P. (1998). Psychometric properties of the 42-item and 21-item versions of the Depression Anxiety Stress Scales (DASS) in clinical groups and a community sample. Psychological Assessment, 10(2), 176-181. http://dx.doi.org/10.1037/1040-3590.10.2.176

Bados, A., Solanas, A. \& Andrés, R. (2005). Psychometric properties of the Spanish version of Depression, Anxiety and Stress Scales (DASS). Psicothema, 17(4), 679-683. Recuperado de http://www. psicothema.com/pdf/3165.pdf

Barco, V., Miranda, Y., Herrera, D. \& Álvarez, Z. (2008). El síndrome de burnout en enfermeros básicos del segundo año de la carrera. Revista cubana de enfermería, 24(3-4). Recuperado de http:// scielo.sld.cu/pdf/enf/v24n3-4/enf05308.pdf

Casal, J. y Mateu,E. (2003). Tipos de muestreos. Rev. Epidem. Med. Prev., (1)3-7. Recuperado de http://www.mat.uson. $\mathrm{mx} / \sim \mathrm{ftapia} /$ Lecturas$\% 20$ Adicionales $\% 20 \% 28 \mathrm{C} \% \mathrm{C} 3 \% \mathrm{~B} 3 \mathrm{mo} \% 20$ dise\%C3\%B1ar\%20una\%20encuesta\%29/TiposMuestreo1.pdf

De Vargas, D. \& Dias, A. P. (2011). Depression prevalence in Intensive Care Unit nursing workers: a study at hospitals in a northwestern city of São Paulo State. Revista Latino-Américana de Enfermagem, 19(5), 1114-1121. http://dx.doi.org/10.1590/ S0104-11692011000500008

Enríquez, C. B., Colunga, C., Preciado, M. L., Ángel, M. \& Domínguez, R. (2011). Factores psicosociales y estrés en trabajo hospitalario de enfermería en Guadalajara, México. Revista Colombiana de Salud Ocupacional, 1(1), 23-26. Recuperado de http://www.revistacolombianadesaludocupacional.co/detallearticulo.php?nroarticulo=7

Flores-Villavicencio, M. E., Troyo-Sanromán, R., Valle, M. A. \& Vega-López, M. G. (2010). Ansiedad y estrés en la práctica del personal de enfermería en un hospital de tercer nivel en Guadalajara. Revista electrónica de Psicología Iztacala, 13(1), 1-17. Recuperado de http://www.iztacala.unam.mx/carreras/psicologia/ psiclin/vol13num1/Art1Vol13No1.pdf

Fonseca-Pedrero, E., Paino, M., Lemos-Giráldez, S. \& Muñiz, J. (2010). Propiedades psicométricas de la depresión, ansiedad y estrés (DASS-21) en universitarios españoles. Revista Ansiedad y Estrés, 16(2-3), 215-226.

Gil-Monte, P. (2010). Situación actual y perspectiva de futuro en el estudio del estrés laboral, Informació psicológica, (100), 68-83. Recuperado de http://dialnet.unirioja.es/servlet/ articulo? codigo $=3642757$

Henry, J. D. \& Crawford, J. R. (2005). The short-form version of the Depression Anxiety Stress Scales (DASS-21): Construct validity and normative data in a large non-clinical sample. British Journal of Clinical Psychology, 44(2), 227-239. Recuperado de http://homepages.abdn.ac.uk/j.crawford/pages/dept/pdfs/ BJCP_2005_DASS21.pdf

Hernández, J. R. (2003). Estrés y Burnout en profesionales de la salud de los niveles primario y secundario de atención. Revista Cubana Salud Pública, 29(2), 103-110. Recuperado de http://scielo.sld. $\mathrm{cu} / \mathrm{pdf} / \mathrm{rcsp} / \mathrm{v} 29 \mathrm{n} 2 / \mathrm{spu} 02203 . \mathrm{pdf}$

Hernández, R. (Mayo, 2005). La depresión en niños como problema de salud pública. Trabajo presentado en el Seminario Permanente de Estudios Prospectivos, Facultad de Ciencias Políticas y Sociales. Universidad Nacional Autónoma de México, México.

Hurtado, D. \& Pereira, F. (2012). El Síndrome de desgaste profesional (Burnout Síndrome): Manifestación de la ruptura de la reciprocidad laboral. Revista Salud Bosque, 2(2), 29-39. Recuperado de 
http://www.uelbosque.edu.co/sites/default/files/publicaciones/ revistas/revista_salud_bosque/volumen2_numero2/sindrome_desgaste_profesional-vol2_num2.pdf

Korman, G. P. \& Sarudiansky, M. (2011). Modelos teóricos y clínicos para la conceptualización y tratamiento de la depresión. Revista Subjetividad y Procesos Cognitivos, 15(1), 119-145. Recuperado de http://www.scielo.org.ar/scielo.php?script=sci_arttext\&pid $=\mathrm{S} 1852-73102011000100005$

Lerma-Martínez, V., Rosales-Arellano, G. V. \&. Gallegos-Alvarado, M. (2009). Prevalencia de ansiedad y depresión en enfermería a cargo del paciente crónico hospitalizado. Revista Comisión Nacional de Arbitraje Médico, 14(1), 5-10. Recuperado de http:// www.conamed.gob.mx/publicaciones/pdf/REVISTA_OCTDIC_2009_supl1.pdf

López, C., Zegarra, A. \& Cuba, V. (2006). Factores asociados al síndrome de Burnout en enfermeras de emergencia del hospital nacional Guillermo Almenara Irigoyen. Revista de Ciencias de la Salud, 1(1), 53-60. Recuperado de http://posgrado.upeu.edu. pe/revista/file/55-63.pdf

Lovibond, S. H. \& Lovibond, P. F. (1995). Manual for the Depression Anxiety Stress Scales. Sydney: Psychology Foundation.

Melgosa, J. (1995). Nuevo estilo de vida. ¡Sin estrés! Madrid: Safeliz.

Martínez, M., Centeno, C., Sanz-Rubiales, A. \& Del Valle, M. L. (2009). Estudio sobre el Síndrome de Burnout en Profesionales de Enfermería de Cuidados Paliativos del País Vasco. Revista de Medicina de la Univdersisda Navarra, 53(1), 3-8. Recuperado de www.unav.es/.../53_1/pdf/estudio-sobre-el-sindrome-de-burnout. $p d f$

Martínez, R., Tuya, L., Martínez, M., Pérez, A. \& Cánovas, A. (2009). El coeficiente de correlación de los rangos de Spearman. Caracterización. Revista habanera de ciencias médicas, 8(2). Recuperado de http://scielo.sld.cu/scielo.php?script=sci_arttext\&pid=S1729519X2009000200017
Miño, A. (2012). Clima organizacional y estrés laboral asistencial (burnout) en profesores de Enseñanza Media: Un estudio correlacional. Revista PsicologíaCientifica.com, 14(9). Recuperado de http://www.psicologiacientifica.com/burnout-profesores-ensenanza-media

Naranjo, M. L. (2009). Una revisión teórica sobre el estrés y algunos aspectos relevantes de éste en el ámbito educativo. Revista Educación, 33(2), 171-190. Recuperado de http://www.redalyc. org/pdf/440/44012058011.pdf

Piqueras, J. A., Martínez, A. E., Ramos, V., Rivero, R., García, L. J. \& Oblitas, L. A. (2008). Ansiedad, Depresión y Salud. Suma Psicológica, 5(1), 43-74. Recuperado de http://publicaciones. konradlorenz.edu.co/index.php/sumapsi/article/view/16/2

Ramis, Y., Torregrosa, M., Viladrich, C. \& Cruz, J. (2010). Adaptación y validación de la versión española de la Escala de Ansiedad Competitiva SAS-2 para deportistas de iniciación. Psicothema 22(4), 1004-1009. Recuperado de http://www.psicothema.com/ pdf/3833.pdf

Sierra, J. C., Ortega, V. \& Zubeidat, I. (2003). Ansiedad, angustia y estrés: tres conceptos a diferenciar. Revista mal-estar e subjetividade/fortaleza, 3(1), 10-59. Recuperado de http://pepsic.bvsalud. org/pdf/malestar/v3n1/02.pdf

Tully, P. J., Zajac, I. T. \& Venning, A. J. (2009). The structure of anxiety and depression in a normative sample of younger and older Australian adolescents. Journal of Abnormal Child Psychology, 37, 717-726. doi:10.1007/s10802-009-9306-4

Vásquez, C. \& Ríos, J.R. (2009). Prevalencia de estrés, ansiedad y depresión en estudiantes de postgrado, Neurol Neurocir Psiquiat., 42(1-4), 7-12

Zlomke, K. R. (2009). Psychometric properties on internet administered versions of Penn State Worry Questionnaire (PSWQ) and Depression, Anxiety, and Stress Scale (DASS). Computers in Human Behavior, 25, 841-843. 
